\title{
The evolution of the Cornell NanoScale Facility and synergies with the semiconductor Industry
}

D. Tennant

D. Tennant, "The evolution of the Cornell NanoScale Facility and synergies with the semiconductor Industry," Proc. SPIE 10958, Novel Patterning Technologies for Semiconductors, MEMS/NEMS, and MOEMS 2019, 1095802 (10 June 2019); doi: 10.1117/12.2517091 


\title{
The Evolution of the Cornell NanoScale Facility and Synergies with the Semiconductor Industry
}

\author{
D.M. Tennant \\ Cornell NanoScale Science and Technology Facility \\ 250 Duffield Hall, Cornell University \\ Ithaca, NY 14853
}

\begin{abstract}
University fabrication facilities that are both open and shared were created to enable far wider access to tools and methods previously only available to faculty at a few select universities. The lab now known as the Cornell NanoScale Science and Technology Facility (CNF), was the first NSF-supported national user facility in this field about 42 years ago. This talk will consider how open and shared facilities have evolved, and how important it has been to both academic and industrial research in the US. Flexibility and measured changes make CNF and its partners in the National Nanotechnology Coordinated Infrastructure (NNCI) even more relevant going forward as we see technical challenges that must be met due to the explosion in interdisciplinary fields. Some of the needs we see for the future, surprisingly, will have many synergies with the semiconductor industry.
\end{abstract}

Keywords: Cornell NanoScale Facility, History of Nanofabrication, NNCI, NNIN, NNUN, NRRFSS

\section{INTRODUCTION}

I had the timely good fortune to hear Prof. Cyrus Mody, science historian currently at Maastricht University, give the keynote address at the Cornell NanoScale Science and Technology Facility (CNF) Annual Meeting [1]. This talk, based on the research of Prof. Mody and his colleague Hyungsub Choi [2,3] provided an overhead view of the changing landscape of federally funded science and how events led to the concept of shared user facilities such as the National Research and Resource Facility for Submicron Structures (NRRFSS) - the predecessor of the CNF. I attempt here to capture some of the key elements of that presentation to help understand how CNF got started. I will then go on to discuss the many changes and accomplishments of the various collective partners that evolved from 1977 to 2019 from the single lab at to Cornell to what is now a network of 16 nodes funded under the National Nanotechnology Coordinated Infrastructure (NNCI) that have an enormous impact on both the research output of the US and the commercialization of academic innovation. I will conclude by recapping some recent trends that have helped us form a strategic vision for CNF that I believe will be synergistic with the semiconductor and other industries.

\section{UNIVERSITY RESEARCH IN SCIENCE AND ENGINEERING IN THE US}

The readers are invited to view the video recording of the recent talk by Prof. Mody, "Becoming a National resource: CNF and Postwar American Academic Research" that is hosted on the CNF website [1] and to read a more comprehensive history of the dynamic between industry and academic research in his book, The Long Arm of Moore's Law: Microelectronics and American Science [4]. What we learn from this work is that prior to WWII almost no federal funding was available to academic researchers. Most of the basic and to a lesser extent, applied research, was funded by universities, industry or foundations. But the importance of defense related science became evident to the military. So in the late-1940s, 1950s, early-1960s military funding spread to campuses but with the main focus being basic research, as the military's principle need was trained scientists. However, classified research was also being funded by the military and being conducted on campuses. In 1959 the launch of Sputnik by the Soviet Union inspired a space race and led to a dramatic increase in scientific funding. The Advanced Research Project Agency (funded by the military) was formed which in turn founded a number of research facilities including the Material Research Labs (MRLs) on many campuses around 1960.

Novel Patterning Technologies for Semiconductors, MEMS/NEMS, and MOEMS 2019,

edited by Martha I. Sanchez, Eric M. Panning, Proc. of SPIE Vol. 10958, 1095802

(c) 2019 SPIE · CCC code: 0277-786X/19/\$18 - doi: 10.1117/12.2517091 
However, during the mid-1960's science oriented protests at Stanford and MIT and elsewhere surfaced the little discussed classified research being funded on campuses. The period from $1965-1970$ led to dissatisfaction from many stakeholders including the military, in the overall model of the research model in the U.S. The Mansfield amendment [5] barred the Defense Department from using its funds "to carry out any research project or study unless such project or study has a direct and apparent relationship to a specific military function." But the military wanted new materials and for academia to work with industry to make these new materials and discoveries available to them for defense systems. At the same time Universities wanted to end classified research. As a result many of the university labs doing military research spun off from universities (SRI, Cornell Aeronautical Lab, Draper, Lincoln Lab) and NSF inherited numerous labs previous started and funded by the military. The spending chart (in constant dollars) in Fig. 1 illustrates some of these key points and shows the volatility in the research funding scene.

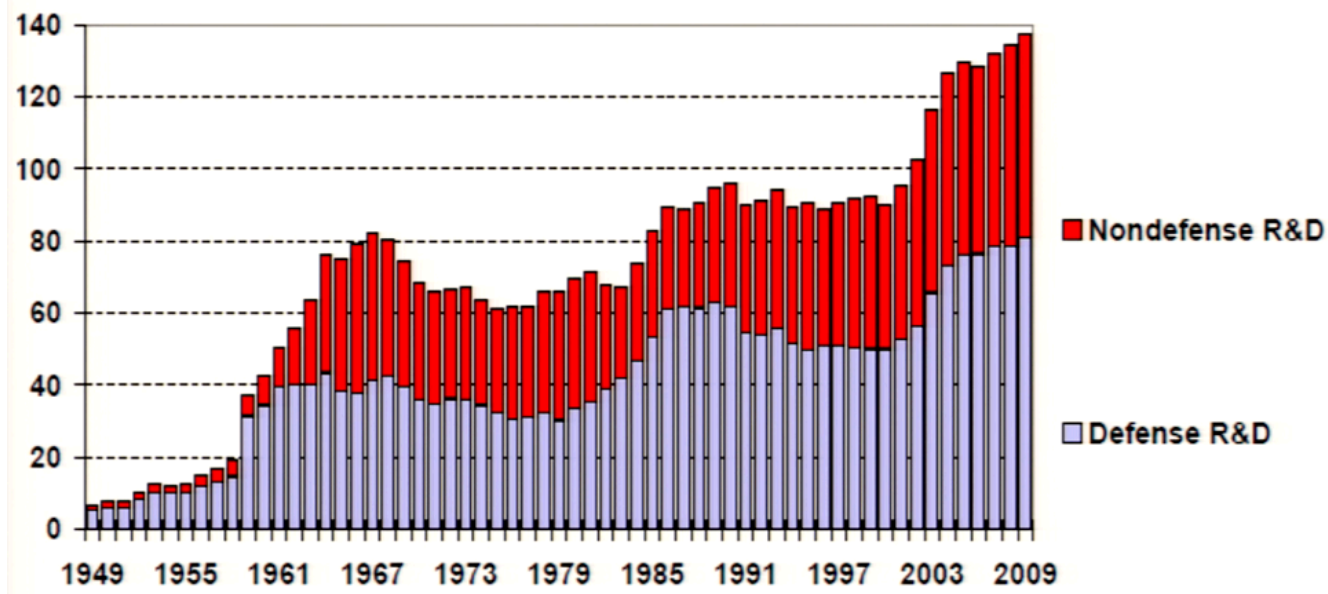

Figure 1. Post WWII R\&D spending for defense and non-defense effort in constant FY2008 dollars. After ref 6.

Around 1969-1970 the space race was considered won and new priorities emerged for science, including; urban plight, the environmental, energy, etc. But around 1975 global competitiveness became the new crisis and among several other industries, microelectronics was a special concern. Internally at NSF there was traction to promote bolstering microelectronics competitiveness but also resistance to have NSF fund electronics where industry and the military already provided significant funding. There was also widespread recognition of a need to narrow the gap between academic and industry research. It was felt that universities couldn't do relevant work on ancient equipment. So there was a heightened need for infrastructure, but also a need to stretch the available funding. This gave rise to the idea of a microstructure focus and the concept that having shared, highly utilized equipment as the solution. During 1976 NSF held three workshops across the country to assess the need and requirements for a university-based national facility. The NSF competition in 1976-7 for a national fabrication facility was a result of these needs, and a milestone for NSF. It was one of the first ventures into starting and sustaining a central facility (remember the MRLs and other centers being operated by NSF for several years, were inherited from the military).

\section{A BRIEF HISTORY NSF FUNDED NANOFACILITIES}

The proposal from Cornell University was led by Prof. Joseph M. Ballantyne, School of Electrical and Computer Engineering at Cornell and NRRFSS was begun in 1977 [7,8]. Dr. Edward D. Wolf became the first permanent NRRFSS director, joining Cornell in 1978. A dedicated building, Knight Lab, a 7,500 square foot clean room, was constructed and occupied in 1981(see Fig. 2). Over the next fifteen years (1982-1997), the facility grew its user community, adding much needed equipment including an electron beam lithography facility. During this period the facility changed in name from 
the National Research and Resource Facility for Submicron Structures to the National Nanofabrication Facility (NNF). Prof. Harold Craighead, School of Applied and Engineering Physics (recruited from Bellcore) became the second director of the facility in 1988 and served until 1995. Prof. Noel C. MacDonald, School of Electrical and Computer Engineering, served as the third director of the facility from 1995 to 1997 followed by Prof. Joseph M. Ballantyne who served as the interim Lester B. Knight Director from 1997 to 1998, when Dr. Sandip Tiwari recruited from IBM, became director (19992006).

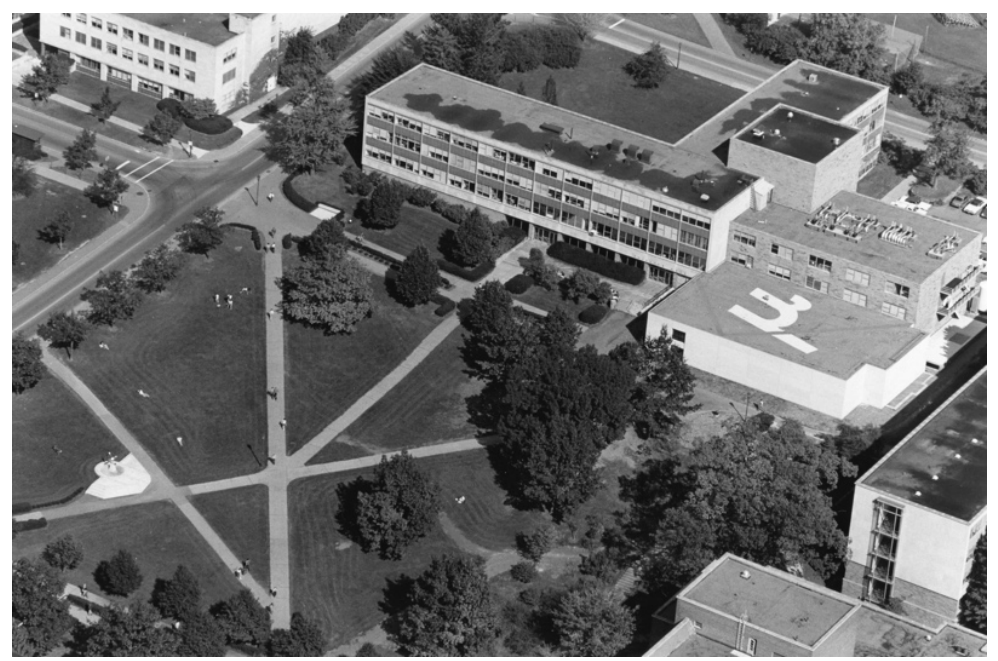

Figure 2. Photo of the engineering quad at Cornell (ca 1981) with the newly built Knight Lab (with the $\mu$ on the roof) that served as the home of the NRRFSS.

In 1993, the concept was expanded to include additional nanofabrication facilities. Cornell University won the competition for the National Nanofabrication Users Network (NNUN) with the Cornell Nanofabrication Facility teaming with four other laboratories; Stanford University, University of California, Santa Barbara (UCSB), The Pennsylvania State University (PSU), and Howard University. NNUN served as a model for national funding of facility networks, and set the stage for an even larger nanotechnology network.

In 2003, a new home for CNF - Duffield Hall, with its 17000 sq. ft. cleanroom and additional non-cleanroom space, was completed, and Cornell University was awarded the National Nanotechnology Infrastructure Network (NNIN) competition. This expanded network included 12 sites; Cornell, Georgia Institute of Technology, Harvard, Howard, PSU, Stanford, UCSB, University of Michigan, University of Minnesota, University of New Mexico, University of Texas at Austin, and the University of Washington. In 2009, the NNIN added Arizona State University and the University of Colorado, and Washington University in St. Louis. During that period, Prof. Tiwari became director of the entire network. In 2006, Prof. John Silcox came on board as interim director until Prof. George Malliaras (2006-2008) took on the role of CNF Director and Don Tennant was hired from Bell Labs as CNF's first Director of Operations and interim Director (20089). Following Malliaras' departure, a new Director, Prof. Dan Ralph (2009-2016) was selected. Overall leadership for the network shifted to Stanford and a new proposal was prepared for renewal of NNIN. Unexpectedly, NSF cancelled the competition, extended the NNIN for one additional year of funding and then held a workshop to plan a new direction for the network. Subsequently a new competition was held for the formation of the National Nanotechnology Coordinated Infrastructure (NNCI) program. Ralph led Cornell's effort to become a member of NNCI. Each member of the NNCI was chosen in an individual review process. The 16 nodes of NNCI are led by Cornell, Arizona State University, Georgia Institute of Technology, University of Pennsylvania, Harvard University, Stanford University, University of Minnesota, University of Texas, and University of Washington, University of California at San Diego, Montana State University, University of Nebraska, Northwestern University, University of Louisville, Virginia Tech, and North Carolina State University. In 2016, the CNF welcomed its newest Director, Professor Christopher Ober, Materials Science and Engineering. 


\section{TECHNOLOGY DEVELOPMENTS AND SYNERGIES WITH MICROELECTRONICS}

The establishment of NRRFSS allowed technologies to be developed by individual research groups in an environment where professional staff could provide an institutional memory and thereby proliferate the techniques developed there.

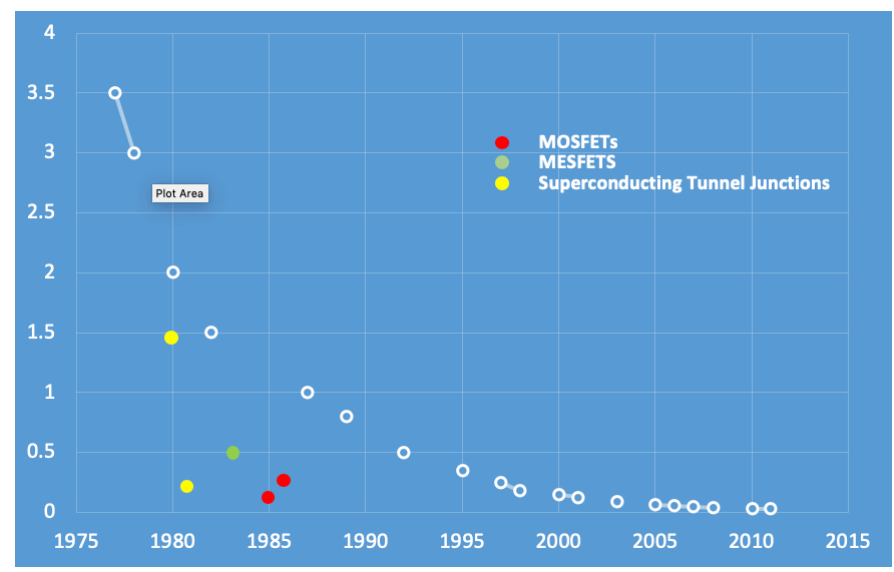

Figure 3. Chart of linewidths used in the manufacture of transistor gates in integrated circuits over the period from 1975 to 2012 (open circles). The added colored dots are research results reported for selected technologies: MOSFETs (red);MESFETs(green); Superconductors (yellow).

Researchers from universities external to Cornell became trained in these methods, thus achieving an important goal of the envisioned shared facility. In many cases academic researchers were able to make significant inroads into the microelectronics field due to access to advanced electronic materials and open access to fabrication facilities. Clearly early adoption of advanced lithography tools, such as electron beam lithography, quickly allowed researchers to access size scales beyond that being used in manufacturing of integrated circuits. Figure 3 plots the critical dimension used in

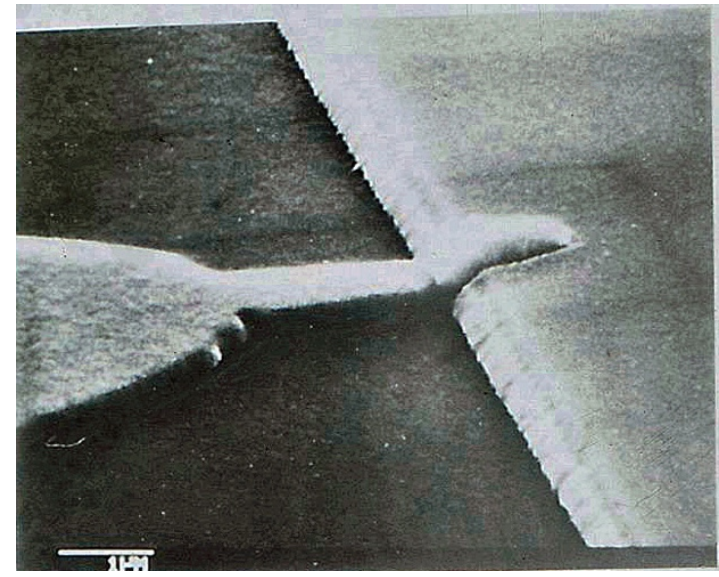

Figure 4. Electron micrograph of a Niobium edge superconducting tunnel junction produced by electron beam lithography at the submicron scale in 1981 by Buhrman, et.al. [ref 9]

IC manufacturing over time. By comparison I have selected a few results reported in the CNF archived research project compendiums for various electronics related achievements. We note that technologies such as transistor gates and superconducting tunnel junctions were being produced at the deep sub-micron size scale well before the IC industry broke the $1 \mu \mathrm{m}$ barrier. This trend continued at CNF and at many other academic facilities and at industry labs (Bell Labs, IBM, H-P, etc.) due to the steady progress in commercially available electron beam lithography. Fig. 4-6 are examples of deep submicron patterning by electron beam lithography as applied to $\mathrm{Nb}$ edge superconducting tunnel junctions (pictured) and S-N-S microbridges (not pictured) [ref 9] ; T-gate technology developed for high frequency III$\mathrm{V}$ semiconductor transistor work [ref 10]; and early $0.1 \mu \mathrm{m}$ gate high electron mobility transistor (HEMT) work [ref 11]. 
From these few examples we can see that early access to advanced lithography and other processing tools in shared facilities started to narrow the gap between industry

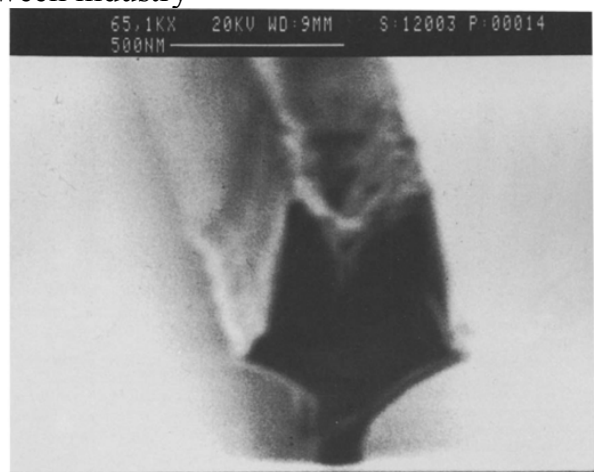

Figure 5. T-Gate structure developed for high frequency transistors using a trilevel electron beam lithography patterning technique. The performance enhancement comes from having a short gate length but a low resistance along the width of the gate. [ref. 10]

and academic research - another important goal of the NSF program. The steady progression of improvements in advanced lithography instruments such as electron beam lithography, in for example, resolution, speed, and precision has been an important differentiator for shared facilities that has enabled world-leading research into microelectronics, MEMS/NEMS, nanophotonics, etc. to take place among university researchers. In parallel with instrument developments such as faster components, better interferometer stages, etc., companies such as FEI and Denka have continued to develop brighter and more stable electron sources that have been incorporated into these tool designs. Furthermore, researchers and facility staff have exploited clever resist schemes [12], new materials [13], and software correction (e.g. proximity effect correction) [14] to allow a wider range of solutions for patterning leading edge devices and structures.

\section{THE EXPANSION INTO INTERDISCIPLINARY RESEARCH}

As observed by Mody [1,4], key conferences like the Gordon Conference on Microfabrication chart the changes in the field over time. The first such conference held in 1976 was dominated by researchers from industrial research labs like IBM Research and Bell Labs and others and was peppered with talk topics that reflected the science and technology of electronic materials, semiconductor-type processing, and resulting devices. In the years following, many of these same individuals moved to academic positions to influence the subjects being studied at universities and new areas like MEMS

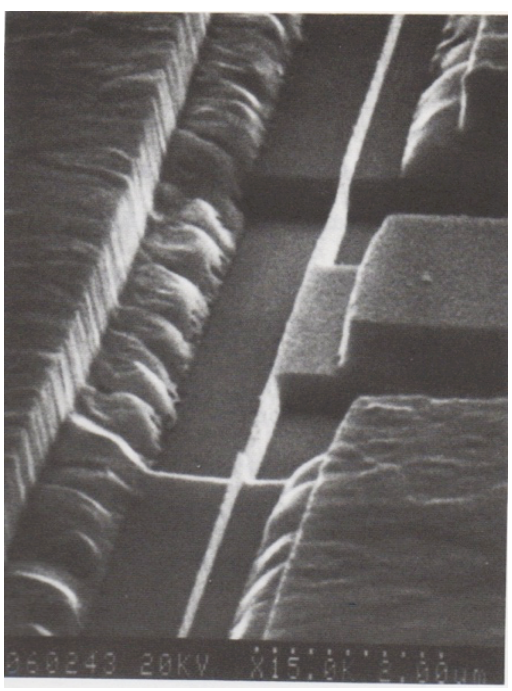

Figure 6. Electron micrograph of a $0.1 \mu \mathrm{m}$ gate length HEMT transistor produced by electrom beam lithography at NRRFSS. [ref. 11] 
emerged to take advantage of the skills and processes developed for microelectronics. But in 1994 the same conference voted to change its name to reflect the "nano" initiative and that year's conference program reflected a far more multi- and inter- disciplinary set of topics. Far more talks on biology, photonics, sensors, catalysis, nanoparticles, optical tweezers, self-assembly, etc. dominated the program. These areas appealed to researchers who by now understood that industrial consortia like SEMATECH and their member companies were handling roadmaps and development of the semiconductor industry's future needs.

One of the key reasons academic labs like CNF at Cornell and labs at MIT, Stanford, UC Berkeley, UCSB, U Michigan, Georgia Tech, and many others, have been able to impact research into so many areas of research - including electronics, is that they were able to access advanced lithography well ahead of similar critical dimensions being available in manufacturing. One important technology that enabled this, is the commercially available electron beam lithography system. Figure 7 illustrates the progress that occurred in electron beam lithography tools over the course of CNF's history. With each acquisition, systems became higher resolution, faster writing speeds, and more precise in their patterning. The Cambridge tool used during NRRFSS could write $0.25 \mu \mathrm{m}$ lines, but at a time when manufacturing done by optical lithography was limited to $1-2 \mu \mathrm{m}$ dimensions. The newest tools can pattern down to the sub-10 $\mathrm{nm}$ regime with deflector

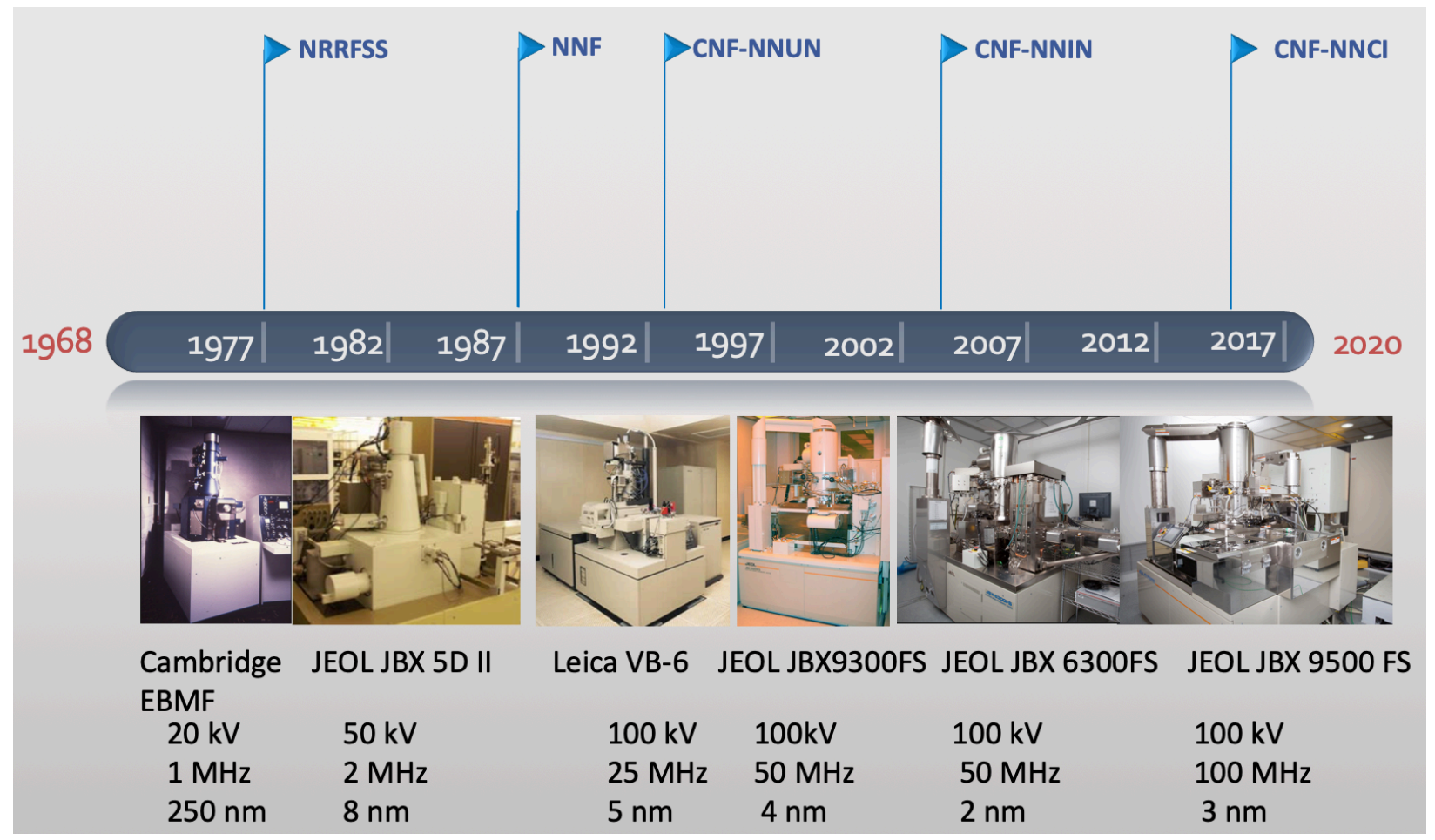

Figure 7. Timeline showing the parallel between the evolution of the shared user facility networks and the acquisition of advanced electron beam lithography tools.

speeds 100 times faster. The newer instruments also include many calibration and correction schemes that allow the electron beam lithography steps to adapt to process related distortions and be used in mix and match ways with other patterning methods.

In addition to the improved instruments, our ability to routinely correct for electron scattering effects via commercial software, improvements in resist materials, and innovations in the use of multilayer resist stacks have allowed researchers to lead technology into new areas such as: $2.5 \mathrm{D}$ and 3D patterning [15], molecular scale probes[16], directed self assembly of block co-polymers [17], silicon nanophotonics [18], integrated optical traps [19], free standing MEMs [20], and many other extremely challenging nanostructure projects. Despite the vastly different capabilities between the modern IC foundry and the typical academic lab, this look-into-the-future capability can directly impact forward looking aspects of 
today's electronics industry. As an example, CNF was key to producing an intentional defect array (with programmed defects down to $5 \mathrm{~nm}$ in size) that was to be used for proving in new wafer inspection systems [21].

Today CNF operates as a node in the NNCI network of university labs open to researchers everywhere. Together the network nodes in aggregate are serving over 13,000 users per year with almost 1900 coming from industry. The network serves 220 academic institutions and over 900 companies and over one million hours of lab time is consumed by its users. CNF users are engaged in hundreds of projects currently, here I describe a few that represent the breadth of projects supported:

Rapid DNA Sequencing - Wanunu and his group from Northeastern University along with scientists from Pacific BioSciences showed that the efficiency of voltage- induced DNA loading into waveguides equipped with nanopores at their floors is five orders of magnitude greater than existing methods [22]. Compared to conventional methods, single molecule, real-time (SMRT) DNA sequencing exhibits longer read lengths than conventional methods, and the ability to read DNA base modifications. However, reading a DNA sequence from sub-ng quantities is impractical due to inefficient delivery of DNA molecules into the confines of zero-mode waveguides, zeptolitre optical cavities in which DNA sequencing proceeds. In addition, they find that DNA loading is nearly length- independent, unlike diffusive loading, which is biased towards shorter fragments. The authors demonstrate here loading and proof-of-principle four-colour sequence readout of a polymerase-bound 20,000 bp long DNA template within seconds from a sub-ng input quantity, a step towards low-input DNA sequencing and mammalian epigenomic mapping of native DNA samples.

Neuroscience - Khodagholy et al., from NYU, and colleagues at Columbia produced a conducting polymer-based conformable microelectrode array (NeuroGrid) and used it to record local field potentials and neural spiking across the dorsal cortical surface of the rat brain, and combined with silicon probe recordings in the hippocampus, to identify candidate physiological patterns [23]. Consolidation of some memories requires hippocampal-neocortical communication. Measurements of this type are made possible with this conformable array that include preamplifiers built in to the flexible electronics.

Self-Folding Graphene Machines - Cohen and McEuen, et. al. at Cornell demonstrated technology using graphene bimorphs that can self-fold to form 3D structures [24]. The group bonded graphene sheets to nanometer-thick layers of glass to produce graphene-glass bimorph actuators that fold to enable the fabrication of 3D structures on the micron scale. The actuators can change shape in less than a second and support the incorporation of embedded electronics. According to the authors, the technology might have applications in the development of miniaturized machines.

Quantum interference between transverse spatial waveguide modes - Lipson et al. at Columbia demonstrated quantum interference between the transverse spatial modes within a single multi-mode waveguide using quantum circuit-building blocks [25]. Integrated quantum optics has the potential to markedly reduce the footprint and resource requirements of quantum information processing systems, but its practical implementation demands broader utilization of the available degrees of freedom within the optical field. This work shows that spatial modes can be controlled to an unprecedented level and have the potential to enable practical and robust quantum information processing.

Microscale Opto-electronically Transduced Electrodes (MOTES) - Molnar et al. at Cornell use the Cornell Nanoscale Facility to demonstrate technology for tetherless neural recording that combines the merits of both optical and electrical modalities in a device $57 \mu \mathrm{m} \times 250 \mu \mathrm{m}$ [26]. While the optics provides means for deep penetration for power transfer as well as signal communication, electronics provides means for efficient use of the delivered optical power for low noise measurements. They identify biological safety as well as fundamental device limits of the proposed techniques, applied engineering safety factors, and made designs accordingly. They have successfully demonstrated that an AlGaAs PVLED can be integrated with commercial CMOS at the back end of the line with minimal post-fabrication and without imposing any unconventional changes on the CMOS fabrication flow. Furthermore, the integrated system was indeed capable of providing low noise measurement of pre-recorded neural signals and being powered remotely.

\section{FUTURE SYNERGIES}

Our vision for CNF continues to evolve as new areas of research take focus to meet national and societal needs. To guide some of our efforts, we have developed a short list of strategic directions on which to develop expertise and make facility 
investments. First, our experience in life sciences over the past two decades leads us to believe that exploiting opportunities to bring greater complexity to chip based biology technologies will be a fruitful strategy. This will take effort to bring together expertise in plant and life sciences with engineering abilities to create integrated modules capable of impacting medical diagnostics, digital agriculture, point of use technologies and the like. Second, we need to develop methods and tooling to support the wave of 2D materials that are being theorized and realized experimentally (graphene, TMDs, etc.) that will provide new classes of materials with emergent properties. This includes a new class of synthesis and fabrication methods that build on traditional strengths. Third, and perhaps most akin to the semiconductor industry, is our goal to acquire tooling and expertise to take heterointegration and CMOS post processing, chip stacking, multi-chip modules, 3D assembly, to the next level. Combining the processing power of mature silicon semiconductor ICs that are widely available from foundries with ready methods of combining these with application specific post processing can make a rich environment for work in AI, medicine, sensing, energy solutions, communications, and many other fields. Last, there is a need to facilitate the "new" wave of quantum information research and development by accruing hardware and baseline processes to support this work as the field converges on a qubit technology. Theses strategic directions, which have their roots in the several decades of research, have the potential to be of mainstream importance to semiconductor companies as they eye the next big thing for their industry.

\section{SUMMARY}

The National Resource and Research Facility for Submicron Structures at Cornell began, in part, to answer the call to arms from international competitiveness in semiconductors and other industries. Its mission, however, was from the start, meant to be broader than just electronics. After $40+$ years and several name changes, CNF is still a vibrant contributor to the U.S. research enterprise, currently as one of 16 sites in the National Nanotechnology Coordinated Infrastructure (NNCI). These geographically distributed laboratories continue to be key enablers for both academic and industry researchers by providing open access to equipment and expert staff at affordable costs. The evolution of CNF from NRRFSS to NNCI (1977-2019) was possible by maintaining flexibility within the facility but also acquiring and developing key technologies, instrumentation, and processes to meet the changing needs. Academic labs like CNF flourish in interdisciplinary research and have also been hugely successful in economic impact. Early work at NRRFSS was successful in making advances in electronics by providing access to lithography (e.g., electron beam systems) and other semiconductor processes at dimensions smaller than generally available in manufacturing, and by opening these tools to many new materials (superconductors, compound semiconductors, and the like). Today these tools continue to be useful to an even broader range of applications and material systems. To continue to anticipate the needs of the community, CNF has developed a strategic vision that is forward looking in life sciences, 2D materials, heterointegration, and quantum Information. It is our hope that our user community can positively impact mainstream industries, such as semiconductors, health care, energy, communications, instrumentation, and digital agriculture to name but a few.

\section{ACKNOWLEDGEMENTS}

This paper was prepared with the support of the Cornell NanoScale Science \& Technology Facility (CNF), a member of the National Nanotechnology Coordinated Infrastructure (NNCI), which is supported by the National Science Foundation (Grant NNCI-1542081). I wish to acknowledge the generous help of Cyrus Mody and the work done by Ed Wolf to document the history of the CNF.

\section{REFERENCES}

[1] Mody C., "Becoming a National resource: CNF and Postwar American Academic Research", talk recorded at the 2018 CNF Annual meeting. Video available at: http://www.cnf.cornell.edu/cnf_2018cnfam_mody.html

[2] Mody C. and Choi H., "From Materials Science to Nanotechnology: Interdisciplinary Center Programs at Cornell University 1960-2000", Historical Studies in the Natural Sciences, Vol. 43, No. 2 (Apr. 1, 2013), pp. 121-161

[3] Choi H. and Mody C., "The Long History of Molecular Electronics: 
Microelectronics Origins of Nanotechnology", Social Studies of Science 39/1 (February 2009) 11-50.

[4] Mody C., The Long Arm of Moore's Law: Microelectronics and American Science, The MIT Press (2017).

[5] see https://www.nsf.gov/nsb/documents/2000/nsb00215/nsb50/1970/mansfield.html

[6] from AAAS, see https://www.aaas.org/sites/default/files/s3fs-public/Function\%253B.jpg

[7] Wolf E., "CNF: A Brief History", written for the CNF 30th Anniversary (2007), Updated by G. Malliaras (2009) and

C. Ober (2016). Archived on the CNF website at: http://www.cnf.cornell.edu/cnf_history.html

[8] Buhrman R.A., Address at the CNF 40th Anniversary Meeting, "CNF- Forty Years and Counting", video available

at: https://vod.video.cornell.edu/media/2017AM-Buhrman/0_shm61qkh

[9] Kleinsasser A., and Buhrman R.A., Appl. Phys. Lett. 37, 841 (1980) for edge junctions, and Warlaumont J., Brown J. C and Buhrman R. A., APL, $34,415,1979$, for SNS microbridges.

[10] Chao P. C., Smith P.M., Wanuga S., Hwang J.C.M., Perkins W.H., Tiberio R., Wolf E.D., "Electron-beam fabrication of quarter-micron T-shaped-gate FETs using a new tri-layer resist system," 1983 International Electron Devices Meeting, Washington, DC, USA, 1983, pp. 613-616.

[11] Chao P.C., Tiberio R.C., Duh K.H.G., Smith P.M., Ballingall J.M., Lester L.F., Lee B.R., Jabra A., Gifford G.G., “0.1 Micron Gate Length Pseudomorphic HEMTs", Electron Dev. Lett, vol. 8 issue 10, 489 (1987).

[12] Carr D.W., Tiberio R.C., "Direct-write Electron Beam Lithography: History and State of the Art", Mat. Res. Soc. Symp. Proc. Vol. 584, 33-43.

[13] for the first use of HSQ as an electron beam resist see: Namatsu H., Yamaguchi T., Nagase M., Yamasaki K., and Kurihara K., Microelectron. Eng. 41-42, 331 (1998).

[14] as an example of commercial proximity correction software, see BEAMER from GenISys at https://genisysgmbh.com/beamer.html

[15] Ray V., Aida Y., Funakoshi R., Kato H., Pang S., J Vac Sci Technol B 30, 06F303 (2012).

[16] Guillorn M. A., Carr D. W., Tiberio R.C., Greenbaum E., Simpson M. L., "Fabrication Of Dissimilar Metal Electrodes With Nanometer Interelectrode Distance For Molecular Electronic Device Characterization,” J. Vac. Sci.

Technol. B 18, 1177 (2000).

[17] Bita I., Yang J., Jung Y., Ross C., Thomas E., Berggren K., Science 321, (2008) 939.

[18] Lipson M., "Guiding, Modulating, and Emitting Light on Silicon-Challenges and Opportunities", Journal Of Lightwave Technology, Vol. 23, No. 12, December 2005.

[19] Lee J.Y., Lu X., Lin Q., "High-Q silicon carbide photonic-crystal cavities," Appl. Phys. Lett. 106, 041106 (2015) [20] Tallur S. and Bhave S. A., "Monolithic 2GHz electrostatically actuated MEMS oscillator with opto-mechanical frequency multiplier," 2013 Transducers \& Eurosensors XXVII: The 17th International Conference on Solid-State Sensors, Actuators and Microsystems (TRANSDUCERS \& EUROSENSORS XXVII), Barcelona, 2013, pp. 1472-1475. [21] Cummings K. D., Bunday B., Malloy M., Hartley J., Banu L., Mellish M., "Patterning of defect arrays with ebeam lithography used to develop a high throughput e-beam defect inspection tool", Journal of Vacuum Science \& Technology B 33, 06FD03 (2015).

[22] Larkin J., Henley R., Jadhav V., Korlach J., Wanunu M., "Length-independent DNA packing into nanopore zeromode waveguides for low-input DNA sequencing", Nature Nanotechnology 12, 1169-1175 (2017).

[23] Khodagholy D., Gelinas J., Buzsaki G., "Learning-enhanced coupling between ripple oscillations in association cortices and hippocampus," Science 20 Oct 2017: Vol. 358, Issue 6361, pp. 369-372

[24] Miskin M. Z., Dorsey K. J., Bircan B., Han Y., Muller D. A., McEuen P. L., and Cohen I., PNAS January 16, 2018115 (3) 466-470

[25] Mohanty A., Zhang M., Dutt A., Ramelow S., Nussenzveig P., Lipson M., "Quantum Interference between transvers spatial waveguide modes", Nature Comm. 8, \#14010 (2017).

[26] Lee S., Cortese A., Gandhi A., Agger E., McEuen P., Molnar A., IEEE Trans Biomedical Circuits and Systems, vol 12 issue 6, $1256-1266$ (2018). 